PROCEEDINGS OF THE

AMERICAN MATHEMATICAL SOCIETY

Volume 128, Number 2, Pages 413-418

S 0002-9939(99)05578-1

Article electronically published on October 12, 1999

\title{
CAUCHY CONDITION FOR THE CONVERGENCE IN CATEGORY
}

\author{
ELŻBIETA WAGNER-BOJAKOWSKA AND WŁADYSŁAW WILCZYŃSKI
}

(Communicated by Franklin D. Tall)

\begin{abstract}
It is well known that the sequence $\left\{f_{n}\right\}_{n \in N}$ of real measurable functions converges in measure to some measurable function $f$ if and only if $\left\{f_{n}\right\}_{n \in N}$ is fundamental in measure.

In this note we introduce the notion of sequence fundamental in category in this manner such that the sequence $\left\{f_{n}\right\}_{n \in N}$ of real functions having the Baire property converges in category to some function $f$ having the Baire property if and only if $\left\{f_{n}\right\}_{n \in N}$ is fundamental in category.
\end{abstract}

Let $(X, S, m)$ be a finite measure space and let $\left\{f_{n}\right\}_{n \in N}$ be a sequence of real $\mathcal{S}$ measurable functions defined on $X$. We say that $\left\{f_{n}\right\}_{n \in N}$ converges in measure to an $\mathcal{S}$-measurable function $f$ if and only if $m\left(\left\{x \in X:\left|f_{n}(x)-f(x)\right| \geq \varepsilon\right\}\right) \underset{n \rightarrow \infty}{\rightarrow} 0$ for each $\varepsilon>0$. We say that $\left\{f_{n}\right\}_{n \in N}$ is fundamental in measure if and only if $m\left(\left\{x \in X:\left|f_{n}(x)-f_{k}(x)\right| \geq \varepsilon\right\}\right) \underset{n, k \rightarrow \infty}{\rightarrow} 0$ for each $\varepsilon>0$. It is well known that the convergence in measure can be described without the notion of measure, using only the $\sigma$-ideal of sets of measure zero. This fact was used in [W] to define the notion of the convergence in category. Recall that $\left\{f_{n}\right\}_{n \in N}$ converges in measure to $f$ if and only if for each subsequence $\left\{f_{n_{m}}\right\}_{m \in N}$ of $\left\{f_{n}\right\}_{n \in N}$ there exists a subsequence $\left\{f_{n_{m_{p}}}\right\}_{p \in N}$ which converges to $f$ a.e. A moment of reflection shows that the fact that $\left\{f_{n}\right\}_{n \in N}$ is fundamental in measure also can be described without the notion of measure. Namely, a sequence $\left\{f_{n}\right\}_{n \in N}$ is fundamental in measure if and only if for all increasing sequences $\left\{n_{k}\right\}_{k \in N}$ and $\left\{m_{k}\right\}_{k \in N}$ of natural numbers the sequence $\left\{f_{n_{k}}-f_{m_{k}}\right\}_{k \in N}$ converges in measure to a function which is a.e. equal to zero. This observation will be used to define the notion of a sequence which is fundamental in category.

Let $X=[0,1]$, let $\mathcal{S}$ be the $\sigma$-algebra of sets having the Baire property and let $\mathcal{I}$ be the $\sigma$-ideal of sets of the first category. We shall say that some property holds $\mathcal{I}$-almost everywhere (abbr. $\mathcal{I}$-a.e.) on $X$ if and only if the set of all points which do not have this property belongs to $\mathcal{I}$. Let $\mathcal{M}$ denote the family of all real,

Received by the editors November 15, 1995 and, in revised form, March 10, 1996.

1991 Mathematics Subject Classification. Primary 28A20, 54A20; Secondary 26A03.

(C)1999 American Mathematical Society 
$\mathcal{S}$-measurable (i.e. having the Baire property), $\mathcal{I}$-a.e. finite and $\mathcal{I}$-a.e. defined on $[0,1]$ functions.

We shall say that a sequence $\left\{f_{n}\right\}_{n \in N}$ of functions from $\mathcal{M}$ converges in category to some function $f \in \mathcal{M}$ if and only if for each subsequence $\left\{f_{n_{m}}\right\}_{m \in N}$ of $\left\{f_{n}\right\}_{n \in N}$ there exists a subsequence $\left\{f_{n_{m_{p}}}\right\}_{p \in N}$ which converges to $f \mathcal{I}$-a.e. We shall use the denotation $f_{n} \underset{n \rightarrow \infty}{\stackrel{\mathcal{I}}{\rightarrow}} f$. We shall say that a sequence $\left\{f_{n}\right\}_{n \in N}$ of functions from $\mathcal{M}$ is fundamental in category if and only if for all increasing sequences of natural numbers $\left\{n_{k}\right\}_{k \in N}$ and $\left\{m_{k}\right\}_{k \in N}$ the sequence $\left\{f_{n_{k}}-f_{m_{k}}\right\}_{k \in N}$ converges in category to a function which is $\mathcal{I}$-a.e. equal to zero.

Theorem 1. If a sequence $\left\{f_{n}\right\}_{n \in N}$ of functions from $\mathcal{M}$ converges to a function $f \in \mathcal{M}$ in category, then $\left\{f_{n}\right\}_{n \in N}$ is fundamental in category.

Proof. Let $\left\{n_{k}\right\}_{k \in N}$ and $\left\{m_{k}\right\}_{k \in N}$ be two increasing sequences of natural numbers. If $\left\{k_{p}\right\}_{p \in N}$ is an increasing sequence of natural numbers, then there exists a subsequence $\left\{k_{p_{r}}\right\}_{r \in N}$ such that $f_{n_{k_{p_{r}}}} \underset{r \rightarrow \infty}{\longrightarrow} f$ except on a set of the first category. For the increasing sequence $\left\{k_{p_{r}}\right\}_{r \in N}$ there exists a subsequence $\left\{k_{p_{r_{s}}}\right\}_{s \in N}$ such that $f_{m_{k_{p_{r}}}} \underset{s \rightarrow \infty}{\rightarrow} f \mathcal{I}$-a.e. Then $f_{n_{k_{p_{r}}}}-f_{m_{k_{p_{r_{s}}}}} \underset{s \rightarrow \infty}{\rightarrow} 0 \mathcal{I}$-a.e., which means that $\left\{f_{n_{k}}-f_{m_{k}}\right\}_{k \in N}$ converges to zero in category.

To prove the inverse theorem first we shall study the behaviour of divergent sequences of functions from $\mathcal{M}$.

Observe that a sequence $\left\{f_{n}\right\}_{n \in N}$ of functions from $\mathcal{M}$ does not converge in category to any function $f \in \mathcal{M}$ if and only if for each $f \in \mathcal{M}$ there exists an increasing sequence $\left\{n_{m}\right\}_{m \in N}$ of natural numbers such that for each subsequence $\left\{n_{m_{p}}\right\}_{p \in N}$ of $\left\{n_{m}\right\}_{m \in N}$ the sequence $\left\{f_{n_{m_{p}}}\right\}_{p \in N}$ does not converge to $f \mathcal{I}$-a.e. The last expression is equivalent to the alternative of the following two conditions:

(1) there exists an increasing sequence $\left\{n_{m}\right\}_{m \in N}$ of natural numbers such that for each subsequence $\left\{n_{m_{p}}\right\}_{p \in N}$ of $\left\{n_{m}\right\}_{m \in N}$ the sequence $\left\{f_{n_{m_{p}}}\right\}_{p \in N}$ does not converge $\mathcal{I}$-a.e. to any function from $\mathcal{M}$,

(2) there exists a pair of functions $g_{1}, g_{2} \in \mathcal{M}$ which are not equivalent (i.e. $\left.\left\{x: g_{1}(x) \neq g_{2}(x)\right\} \notin \mathcal{I}\right)$ and a pair of increasing sequences $\left\{n_{k}\right\}_{k \in N}$ and $\left\{m_{k}\right\}_{k \in N}$ of natural numbers such that $f_{n_{k}} \underset{k \rightarrow \infty}{\rightarrow} g_{1} \mathcal{I}$-a.e. and $f_{m_{k}} \underset{k \rightarrow \infty}{\rightarrow} g_{2} \mathcal{I}$-a.e.

Observe also that for the sequence $\left\{n_{m}\right\}_{m \in N}$ described in (1) we have two possibilities:

(1a) for each increasing subsequence $\left\{n_{m_{p}}\right\}_{p \in N}$ of $\left\{n_{m}\right\}_{m \in N}$

$$
\left\{x \in X: \limsup _{p} f_{n_{m_{p}}}(x)-\liminf _{p} f_{n_{m_{p}}}(x)>0\right\} \notin \mathcal{I}
$$

or, what amounts to the same,

$$
\left\{x \in X: \limsup _{p} f_{n_{m_{p}}}(x)-\liminf _{p} f_{n_{m_{p}}}(x)>\delta\right\} \notin \mathcal{I}
$$

for some $\delta>0$,

(1b) there exists a subsequence $\left\{n_{m_{p}}\right\}_{p \in N}$ of $\left\{n_{m}\right\}_{m \in N}$ for which

$$
\left\{x \in X: \lim _{p \rightarrow \infty} f_{n_{m_{p}}}(x)=+\infty\right\} \cup\left\{x \in X: \lim _{p \rightarrow \infty} f_{n_{m_{p}}}(x)=-\infty\right\} \notin \mathcal{I} .
$$

When (1a) is fulfilled we shall say that a sequence $\left\{f_{n_{m_{p}}}\right\}_{p \in N}$ diverges by oscillating (it means that no subsequence converges $\mathcal{I}$-a.e.; see $[\mathrm{BG}]$ ). 
Observe that all above remarks remain true for arbitrary $\sigma$-algebra $\mathcal{S}$ and $\sigma$-ideal $\mathcal{I} \subset \mathcal{S}$.

For infinite subsets $M, M^{\prime}$ of the set of all natural numbers, $M \subset^{*} M^{\prime}$ will mean that $M-M^{\prime}$ is a finite set.

Replacing the expressions "a.e.", "measure zero" and "measurability" by "except on a set of the first category", "first category" and "the Baire property" in the proofs of Proposition 2.4, Proposition 2.5, Theorem 2.6 and Corollary 2.7 from [BG], we obtain the following lemma.

Lemma 1. Let $\left\{f_{n}\right\}_{n \in N}$ be a sequence of functions from $\mathcal{M}$ such that each subsequence $\left\{f_{n_{m}}\right\}_{m \in N}$ of $\left\{f_{n}\right\}_{n \in N}$ diverges by oscillating. Then there exist a set $Y \subset[0,1]$ of the second category and having the Baire property, a pair of numbers $r \in R$ and $\delta>0$ and an infinite set $M \subset N$ such that for each infinite set $L \subset^{*} M$ and for each set $A \subset Y$, which has the Baire property and is of the second category, there exist a pair of points $x, y \in A$ and an infinite set $L_{0} \subset L$ such that $f_{n}(x)>r+\delta$ and $f_{n}(y)<r$ for each $n \in L_{0}$.

Lemma 2. Let $\left\{f_{n}\right\}_{n \in N}$ be a sequence of functions from $\mathcal{M}$ such that each subsequence $\left\{f_{n_{m}}\right\}_{m \in N}$ of $\left\{f_{n}\right\}_{n \in N}$ diverges by oscillating. Then there exist two increasing sequences $\left\{n_{k}\right\}_{k \in N}$ and $\left\{m_{k}\right\}_{k \in N}$ of natural numbers such that $\left\{f_{n_{k}}-f_{m_{k}}\right\}_{k \in N}$ does not converge to zero in category.

Proof. Let $P_{n} \subset[0,1]$ for $n \in N$ be a set of the first category such that $f_{n} \mid[0,1]-P_{n}$ is continuous (compare [O], th. 8.1).

Let $Y, r, \delta$ and $M$ be as in Lemma 1. We can obviously suppose that $Y \subset$ $[0,1]-\bigcup_{n=1}^{\infty} P_{n}$, so all functions are continuous at each point of $Y$. Let $(a, b) \subset[0,1]$ be an interval such that $(a, b)-Y \in \mathcal{I}$ (recall that $Y \in \mathcal{S}-\mathcal{I}$ ). Observe that if $(c, d) \subset(a, b)$, then also $(c, d)-Y \in \mathcal{I}$.

Put $A=(a, b) \cap Y$. From Lemma 1 there exist an infinite set $M_{1}^{1} \subset M$ and a point $x_{1}^{1} \in A$ such that $f_{n}\left(x_{1}^{1}\right)>r+\delta$ for $n \in M_{1}^{1}$. Choose $n_{1} \in M_{1}^{1}$. From the continuity of $f_{n_{1}}$ at $x_{1}^{1}$ on $Y$ it follows that there exists $\eta_{1}>0$ such that $f_{n_{1}}(x)>r+\delta$ for $x \in\left(x_{1}^{1}-\eta_{1}, x_{1}^{1}+\eta_{1}\right) \cap Y$. Now for $A=\left(x_{1}^{1}-\eta_{1}, x_{1}^{1}+\eta_{1}\right) \cap Y$ and $M_{1}^{1}$ again by virtue of Lemma 1 there exist an infinite set $M_{2}^{1} \subset M_{1}^{1}$ and a point $y_{1}^{1} \in A$ such that $f_{n}\left(y_{1}^{1}\right)<r$ for $n \in M_{2}^{1}$. Choose $m_{1} \in M_{2}^{1}$. From the continuity of $f_{m_{1}}$ at $y_{1}^{1}$ on $Y$ it follows that there exists $\varepsilon_{1}>0$ such that $f_{m_{1}}(x)<r$ for $x \in\left(y_{1}^{1}-\varepsilon_{1}, y_{1}^{1}+\varepsilon_{1}\right) \cap Y \subset\left(x_{1}^{1}-\eta_{1}, x_{1}^{1}+\eta_{1}\right) \cap Y$. Let $G_{1}=\left(y_{1}^{1}-\varepsilon_{1}, y_{1}^{1}+\varepsilon_{1}\right)$. Then we have $f_{n_{1}}(x)-f_{m_{1}}(x)>\delta$ on $G_{1} \cap Y$.

Suppose that we have chosen increasing finite sequences $\left(n_{1}, \ldots, n_{k-1}\right)$ and $\left(m_{1}, \ldots, m_{k-1}\right)$ and a sequence $\left(G_{1}, \ldots, G_{k-1}\right)$ of open sets such that

$$
G_{j} \cap\left(a+\frac{i-1}{j}(b-a), a+\frac{i}{j}(b-a)\right) \neq \emptyset
$$

for $j \in\{1, \ldots, k-1\}$ and $i \in\{1, \ldots, j\}$ and $f_{n_{j}}(x)-f_{m_{j}}(x)>\delta$ for $x \in G_{j} \cap Y$, $j \in\{1, \ldots, k-1\}$. We have also chosen descending sequences of infinite sets of natural numbers $M_{1}^{1} \supset M_{2}^{1} ; M_{1}^{2} \supset \ldots \supset M_{4}^{2} ; \ldots ; M_{1}^{k-1} \supset \ldots \supset M_{2(k-1)}^{k-1}$.

To make an inductive step we divide $(a, b)$ into $k$ parts $\left\{\left(a_{i}^{k}, b_{i}^{k}\right)\right\}_{i=1, \ldots, k}$ of equal length, i.e. such that $a_{i}^{k}=a+\frac{i-1}{k}(b-a), b_{i}^{k}=a+\frac{i}{k}(b-a)$. For $A=\left(a_{1}^{k}, b_{1}^{k}\right) \cap Y$ and $M$ we use Lemma 1 to find an infinite set $M_{1}^{k} \subset M$ and a point $x_{1}^{k} \in A$ such that $f_{n}\left(x_{1}^{k}\right)>r+\delta$ for $n \in M_{1}^{k}$. Then we repeat the procedure for $A=\left(a_{2}^{k}, b_{2}^{k}\right) \cap Y$ and $M_{1}^{k}$ obtaining $x_{2}^{k} \in A$ and an infinite set $M_{2}^{k} \subset M_{1}^{k}$ such that $f_{n}\left(x_{2}^{k}\right)>r+\delta$ 
for $n \in M_{2}^{k}$ (observe that also $f_{n}\left(x_{1}^{k}\right)>r+\delta$ for $n \in M_{2}^{k}$ ). After $k$ choices we obtain an infinite set $M_{k}^{k}$ of natural numbers and a set $\left\{x_{1}^{k}, \ldots, x_{k}^{k}\right\} \subset Y$ such that $x_{1}^{k} \in\left(a_{i}^{k}, b_{i}^{k}\right)$ and $f_{n}\left(x_{i}^{k}\right)>r+\delta$ for $i \in\{1, \ldots, k\}$ and $n \in M_{k}^{k}$.

Choose $n_{k} \in M_{k}^{k}$ such that $n_{k}>n_{k-1}$. From the continuity of $f_{n_{k}}$ at each $x_{i}^{k}$, $i \in\{1, \ldots, k\}$, on $Y$ it follows that there exists $\eta_{k}>0$ such that $f_{n_{k}}(x)>r+\delta$ for $x \in \bigcup_{i=1}^{k}\left(x_{i}^{k}-\eta_{k}, x_{i}^{k}+\eta_{k}\right) \cap Y$.

To find $m_{k}$ and the open set $G_{k}$ we proceed as follows: for $A=\left(x_{1}^{k}-\eta_{1}, x_{1}^{k}+\right.$ $\left.\eta_{1}\right) \cap\left(a_{1}^{k}, b_{1}^{k}\right) \cap Y$ and $M_{k}^{k}$ applying Lemma 1 we choose $y_{1}^{k} \in A$ and an infinite set $M_{k+1}^{k} \subset M_{k}^{k}$ such that $f_{n}\left(y_{1}^{k}\right)<r$ for $n \in M_{k+1}^{k}$. Then we choose successively infinite sets of natural numbers $M_{k+2}^{k} \supset M_{k+3}^{k} \supset \ldots \supset M_{2 k}^{k}$ (of course, $M_{k+2}^{k} \subset$ $\left.M_{k+1}^{k}\right)$ and points $y_{2}^{k}, \ldots, y_{k}^{k}$ such that $y_{i}^{k} \in\left(x_{i}^{k}-\eta_{k}, x_{i}^{k}+\eta\right) \cap\left(a_{i}^{k}, b_{i}^{k}\right) \cap Y$ for $i \in\{2, \ldots, k\}$ and $f_{n}\left(y_{i}^{k}\right)<r$ for $n \in M_{k+i}^{k}$. At last, we choose $m_{k}>m_{k-1}$ from the set $M_{2 k}^{k}$. Then we have $f_{m_{k}}\left(y_{i}^{k}\right)<r$ for $i \in\{1, \ldots, k\}$. From the continuity of $f_{m_{k}}$ at each $y_{i}^{k}, i \in\{1, \ldots, k\}$, it follows that there exists $\varepsilon_{k}>0$ such that $\left(y_{i}^{k}-\varepsilon_{k}, y_{i}^{k}+\varepsilon_{k}\right) \subset\left(x_{i}^{k}-\eta_{k}, x_{i}^{k}+\eta\right)$ and if we put $G_{k}=\bigcup_{i=1}^{k}\left(y_{i}^{k}-\varepsilon_{k}, y_{i}^{k}+\varepsilon_{k}\right)$, then $f_{m_{k}}(x)<r$ for $x \in G_{k} \cap Y$. Then we have $f_{n_{k}}(x)-f_{m_{k}}(x)>\delta$ for $x \in G_{k} \cap Y$ and $G_{k} \cap\left(a+\frac{i-1}{k}(b-a), a+\frac{i}{k}(b-a)\right) \neq \emptyset$ for $i \in\{1, \ldots, k\}$.

Since for each increasing sequence $\left\{k_{p}\right\}_{p \in N}$ of natural numbers we have $E=$ $\left\{x \in[0,1]: \lim \sup _{p}\left(f_{n_{k_{p}}}(x)-f_{m_{k_{p}}}(x)\right) \geq \delta\right\} \supset \bigcap_{j=1}^{\infty} \bigcup_{p=j}^{\infty}\left\{x \in[0,1]: f_{n_{k_{p}}}(x)-\right.$ $\left.f_{m_{k_{p}}}(x)>\delta\right\} \supset Y \cap \bigcap_{j=1}^{\infty} \bigcup_{p=j}^{\infty} G_{k_{p}}$ and, as is not difficult to see, the set $\bigcup_{p=j}^{\infty} G_{k_{p}}$ is open and dense in $(a, b)$ for each $j \in N$, so $E$ is residual in $(a, b)$ and hence $\left\{f_{n_{k_{p}}}-f_{m_{k_{p}}}\right\}_{p \in N}$ does not converge $\mathcal{I}$-a.e. Finally, $\left\{f_{n_{k}}-f_{m_{k}}\right\}_{k \in N}$ does not converge in category.

Theorem 2. If a sequence $\left\{f_{n}\right\}_{n \in N}$ of functions from $\mathcal{M}$ is fundamental in category, then it converges in category to some function $f \in \mathcal{M}$.

Proof. Suppose that $\left\{f_{n}\right\}_{n \in N}$ does not converge in category to any function from $\mathcal{M}$. Then, according to remarks preceding the series of lemmas, either (1) there exists a pair of functions $g_{1}, g_{2} \in \mathcal{M}$ which are not equivalent and a pair of increasing sequences $\left\{n_{k}\right\}_{k \in N}$ and $\left\{m_{k}\right\}_{k \in N}$ of natural numbers such that $f_{n_{k}} \underset{k \rightarrow \infty}{\rightarrow} g_{1}$ $\mathcal{I}$-a.e. and $f_{m_{k}} \underset{k \rightarrow \infty}{\rightarrow} g_{2} \mathcal{I}$-a.e., or (2) there exists an increasing sequence $\left\{n_{m}\right\}_{m \in N}$ of natural numbers such that for each subsequence $\left\{n_{m_{p}}\right\}_{p \in N}$ of $\left\{n_{m}\right\}_{m \in N}$ the sequence $\left\{f_{n_{m_{p}}}\right\}_{p \in N}$ diverges by oscillating, or (3) there exists an increasing sequence $\left\{n_{m}\right\}_{m \in N}$ of natural numbers such that $\left\{x \in[0,1]: \lim _{m \rightarrow \infty} f_{n_{m}}(x)=+\infty\right\} \notin \mathcal{I}$ or $\left\{x \in[0,1]: \lim _{m \rightarrow \infty} f_{n_{m}}(x)=-\infty\right\} \notin \mathcal{I}$. In case (1) the sequence $\left\{f_{n}\right\}_{n \in N}$ is not fundamental in category because $\left\{f_{n_{k}}-f_{m_{k}}\right\}_{k \in N}$ does not converge to zero in category. In case (2) from Lemma 2 we obtain immediately that $\left\{f_{n}\right\}_{n \in N}$ is not fundamental in category. Suppose now that in case (3) the first possibility holds. To simplify the description assume that $\left\{n_{m}\right\}_{m \in N}$ is the sequence $\{n\}_{n \in N}$ of all natural numbers, i.e. that $E=\left\{x \in[0,1]: \lim _{n \rightarrow \infty} f_{n}(x)=+\infty\right\} \notin \mathcal{I}$. Since $E=$ $\bigcap_{k=1}^{\infty} \bigcup_{m=1}^{\infty} \bigcap_{n=m}^{\infty}\left\{x \in[0,1]: f_{n}(x) \geq k\right\}$, the set $E$ has the Baire property and there exists an interval $(a, b)$ such that $(a, b)-E \in \mathcal{I}$. Take $Y=(a, b) \cap E-\bigcup_{n=1}^{\infty} P_{n}$, where, as before, $P_{n} \in \mathcal{I}$ is a set such that $f_{n} \mid[0,1]-P_{n}$ is continuous. We shall find an increasing sequence $\left\{j_{n}\right\}_{n \in N}$ of natural numbers such that $\left\{f_{j_{n}}-f_{n}\right\}_{n \in N}$ does not converge to zero in category. Put $j_{1}=1$. Suppose that we have constructed $j_{n-1}$. To find $j_{n}$ choose an increasing sequence $x_{1}^{n}<x_{2}^{n}<\ldots<x_{k_{n}}^{n}$ such that 
$x_{i}^{n} \in Y$ for $i \in\left\{1, \ldots, k_{n}\right\}, x_{1}^{n}-a<\frac{1}{n}, b-x_{k_{n}}^{n}<\frac{1}{n}$ and $x_{i}^{n}-x_{i-1}^{n}<\frac{1}{n}$ for $i \in\left\{2, \ldots, k_{n}\right\}$.

Let $c_{n}=\max \left\{f_{n}\left(x_{1}^{n}\right), \ldots, f_{n}\left(x_{k_{n}}^{n}\right)\right\}$. Since $\left\{f_{n}(x)\right\}_{n \in N}$ diverges to $+\infty$ at each point $x \in Y$, there exists a natural number $j_{n}>j_{n-1}$ such that

$$
\min \left\{f_{j_{n}}\left(x_{1}^{n}\right), \ldots, f_{j_{n}}\left(x_{k_{n}}^{n}\right)\right\}>c_{n}+1 .
$$

From the continuity of all $f_{n}$ 's on $Y$ we conclude that there exists an open set $G_{n} \supset\left\{x_{1}^{n}, \ldots, x_{k_{n}}^{n}\right\}$ such that $f_{j_{n}}(x)-f_{n}(x)>1$ for all $x \in G_{n} \cap Y$. Hence for each increasing sequence $\left\{n_{p}\right\}_{p \in N}$ of natural numbers we have $\{x \in(a, b)$ : $\left\{f_{j_{n_{p}}}(x)-f_{n_{p}}(x)\right\}_{p \in N}$ does not converge to zero $\} \supset Y \cap \lim \sup _{p} G_{n_{p}}$. It is not difficult to see that the last set is residual in $(a, b)$. Hence $\left\{f_{j_{n}}-f_{n}\right\}_{n \in N}$ does not converge to zero in category, so $\left\{f_{n}\right\}_{n \in N}$ is not fundamental in category.

Suppose now that $X=[0,1], \mathcal{S}_{1}$ is the $\sigma$-algebra of measurable sets having the Baire property, $\mathcal{I}_{1}$ is the $\sigma$-ideal of null sets of the first category and $\mathcal{M}_{1}$ is the family of all real, $\mathcal{S}_{1}$-measurable, $\mathcal{I}_{1}$-a.e. finite and $\mathcal{I}_{1}$-a.e. defined on $[0,1]$ functions. The notion of the convergence with respect to $\mathcal{I}_{1}$ as well as the notion of fundamentality in $\mathcal{I}_{1}$ are defined in the natural way. The proof of the following theorem is exactly the same as the proof of Theorem 1 :

Theorem 1a. If a sequence $\left\{f_{n}\right\}_{n \in N}$ of functions from $\mathcal{M}_{1}$ converges to a function $f \in \mathcal{M}_{1}$ with respect to $\mathcal{S}_{1}$, then $\left\{f_{n}\right\}_{n \in N}$ is fundamental in $\mathcal{S}_{1}$.

To prove the inverse theorem observe that if $[0,1]=A \cup B$, where $A$ is a null set and $B$ is a set of the first category, then a set $E \subset[0,1]$ is a null set of the first category if and only if $E \cap A$ is of the first category and $E \cap B$ is a null set.

Theorem 2a. If a sequence $\left\{f_{n}\right\}_{n \in N}$ of functions from $\mathcal{M}_{1}$ is fundamental in $\mathcal{I}_{1}$, then it converges with respect to $\mathcal{I}_{1}$ to some function $f \in \mathcal{M}_{1}$.

Proof. Let $[0,1]=A \cup B$, where $A$ is a null set, $B$ is a set of the first category and $A \cap B=\emptyset$. Put for each $n \in N$

$$
g_{n}(x)= \begin{cases}f_{n}(x) & \text { for } x \in A \\ 0 & \text { for } x \in B\end{cases}
$$

and

$$
h_{n}(x)= \begin{cases}f_{n}(x) & \text { for } x \in B \\ 0 & \text { for } x \in A\end{cases}
$$

Then a sequence $\left\{g_{n}\right\}_{n \in N}$ consists of functions from $\mathcal{M}$ and is fundamental in category, so by virtue of Theorem 2 it converges to some function $g \in \mathcal{M}$. Also $\left\{h_{n}\right\}_{n \in N}$ is a sequence of measurable functions fundamental in measure, so it converges to some measurable function $h$.

Taking into account the above observation, it is not difficult to see that a sequence $\left\{f_{n}\right\}_{n \in N}$ converges in $\mathcal{I}_{1}$ to a function $f=(g \mid A) \cup(h \mid B)$. 
In $[\mathrm{V}]$ one can find an example of partially ordered space, in which (o)-fundamental sequences need not converge. However, the pair $(\mathcal{S}, \mathcal{I})$ in $[\mathrm{V}]$ does not fulfil ccc.

Question. Is it possible to find a $\sigma$-algebra $\mathcal{S}$ and a $\sigma$-ideal $\mathcal{I} \subset \mathcal{S}$ of subsets of $[0,1]$ such that the pair $(\mathcal{S}, \mathcal{I})$ fulfils ccc and there exists a sequence $\left\{f_{n}\right\}_{n \in N}$ of real $\mathcal{S}$-measurable $\mathcal{I}$-a.e. finite and $\mathcal{I}$-a.e. defined on $[0,1]$ functions which is fundamental with respect to $\mathcal{I}$ but does not converge with respect to $\mathcal{I}$ ?

\section{REFERENCES}

[BG] D. Bucchioni, A. Goldman, Sur la convergence presque partout des suites de fonctions mesurables, Can. J. Math. 30 (1978), 638-649. MR 81e:28001

[O] J.C. Oxtoby, Measure and Category, Springer Verlag, New York, 1971. MR 52:14213

[V] D. Vladimirov, On the completeness of partially ordered space, Uspeckhi Mat. N. 15, 2 (92) (1960), 165-172. MR 22:4933

[W] E. Wagner, Sequences of measurable functions, Fund. Math. 112 (1981), 89-102. MR 82m:28010

Faculty of Mathematics, Łódź University, ul. Stefana Banacha 22, 90-238 Łódź, POLAND

E-mail address: wagner@krysia.uni.lodz.pl

E-mail address: wwil@krysia.uni.lodz.pl 